\title{
Correction to: Sleep and physical activity: a cross-sectional objective profile of people with rheumatoid arthritis
}

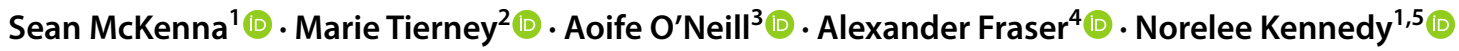

Published online: 5 September 2018

○) Springer-Verlag GmbH Germany, part of Springer Nature 2018

\section{Correction to: \\ Rheumatology International (2018) 38:845-853 \\ https://doi.org/10.1007/s00296-018-4009-1}

One of the reference entries, Ref. [45], in the published article was incomplete. The complete details are provided below:
45. McKenna S, Donnelly A, Fraser A, Comber L, Kennedy N (2017) Does exercise impact on sleep for people who have rheumatoid arthritis? A systematic review. Rheumatol Int 37:963-974. https://doi.org/10.1007/s00296-017-3681-x
The original article can be found online at https://doi.org/10.1007/ s00296-018-4009-1.

\section{Sean McKenna}

sean.g.mckenna@ul.ie

Marie Tierney

marie.m.tierney@nuig.ie

Aoife O’Neill

aoife.oneill@ul.ie

Alexander Fraser

alexander.fraser@hse.ie

Norelee Kennedy

Norelee.kennedy@ul.ie
1 School of Allied Health, Discipline of Physiotherapy, University of Limerick, Limerick, Ireland

2 Discipline of General Practice, National University of Galway, Galway, Ireland

3 Department of Mathematics and Statistics, University of Limerick, Limerick, Ireland

4 Department of Rheumatology, University Hospitals Limerick, Limerick, Ireland

5 Health Research Institute (HRI), UL Hospitals Clinical Research Unit (CRU), University of Limerick, Limerick, Ireland 